\title{
Dopaminergic contributions to distance estimation in Parkinson's disease: A sensory-perceptual deficit?
}

\author{
by \\ Kaylena Ehgoetz Martens \\ A thesis \\ presented to the University of Waterloo \\ in fulfillment of the \\ thesis requirement for the degree of \\ Master of Arts \\ in \\ Psychology
}

Waterloo, Ontario, Canada, 2012

CKaylena Ehgoetz Martens 2012 


\section{AUTHOR'S DECLARATION}

I hereby declare that I am the sole author of this thesis. This is a true copy of the thesis, including any required final revisions, as accepted by my examiners.

I understand that my thesis may be made electronically available to the public. 


\begin{abstract}
Recent research has found that perceptual deficits exist in Parkinson's disease (PD), yet the link between perception and movement impairments is not well understood. Inaccurate estimation of distance has the potential to be an underlying cause of movement impairments. Alternatively, those with PD may not be able to perceive their own movements accurately. The main objective of this thesis was to evaluate (1) whether distance estimation is influenced by static perception compared to perception during movement in $\mathrm{PD}$, (2) how visual motion processing contributes to distance estimation during movement, and (3) how dopaminergic medication contributes to these distance estimation deficits. Thirty-seven participants (19 individuals with PD, 18 age-matched healthy control participants (HC) estimated distance to a remembered target in a total of 48 trials, in 4 randomized blocks. Estimation conditions included: (i) no motion: participants pointed with a laser, (ii) motion: participants walked to the estimated position, (iii) visual motion (wheelchair): participants were pushed in a wheelchair while they gave their estimate, (iv) visual motion (VR): participants completed their distance estimate while seated and viewed themselves (as if they were walking) in VR. PD patients completed this protocol twice; once OFF and once ON dopaminergic medication. Participants were matched for age, distance acuity, Modified Mini Mental State Exam (3MS), spatial working memory and motor planning ability. In Study 1 (no motion vs. motion), individuals with PD and healthy control participants did not differ in judgment accuracy during the no motion condition. However, those with PD did have greater amounts of error compared to healthy control participants while estimating distance during the motion condition. Similarly, those with PD significantly underestimated the target position compared to healthy control participants during the motion condition only. Individuals with PD demonstrated greater variability overall. In Study 2, error did not differ between PD and HC groups during visual motion perception (wheelchair). Interestingly, the HC group tended to perform significantly worse than those with PD in the VR condition.
\end{abstract}


Overall, across both studies there was no significant influence of dopaminergic medication in any of the conditions. Individuals with PD demonstrated distance estimation deficits only when required to move through their environment. In contrast to estimations made with movement, neither static estimation nor estimations made with visual motion revealed significant differences between the two groups. Thus perceptual estimation deficits appear to occur only during movement, which may be suggestive of an underlying sensory processing deficit which leads to a problem integrating vision and self-motion information. 


\section{Acknowledgements}

It is a pleasure to thank the many people who made this thesis possible.

I would like to take this opportunity to extend a special thanks to my supervisors, Colin Ellard and Quincy Almeida, who dedicated their time and efforts into this thesis. I admire their enthusiasm for research and their continual commitment to their students' success.

I would also like to thank my fellow lab mates. I am very fortunate to be a part of such a knowledgeable research team that is exceptionally supportive and eager to provide assistance whenever possible.

To the patients and study participants, I cannot express my appreciation for their willingness to participate in my research. They are the inspirations behind this thesis, and I am very grateful for the time they have invested in our Centre and my research. This thesis could not have been done without their continual commitment and cooperation to the MDRC.

I would like to thank Mike Dixon and James Danckert for their insightful comments on the design and presentation of this research.

Finally, I would like to thank my family and friends who have helped me through the stress I have experienced in completing this thesis. I am so lucky to have such remarkable people in my life. Specifically, I would like to thank my brothers, Leahm, Riley and Brayden, for offering their help wherever possible and always being an outlet to talk to. I would like to express my deepest gratitude to my parents for their understanding and continual encouragement, and I would also like to thank my fiancé Brett, for his never ending positivity, motivation, love and support.

Thank you to everyone I have mentioned, and the many I have failed to mention. This document is the result of the contributions from all of you! 


\section{Dedication}

I would like to dedicate this thesis to my parents. First, to my mother, for her love, support and understanding. She has inspired me to pursue my passions and dreams and always offers continual support and loving words to help me get through stressful times. To my father, who has taught me to work hard and always put forth my highest level of effort. I would like to thank you both for giving me the opportunity to expand my knowledge in university, and teaching me to persevere tough times and struggles that may stand in the way of my goals. Thank you for loving me, providing for me every opportunity possible and teaching me the discipline to achieve anything I aspire to. This thesis and any success I achieve is a testament to your hard work as loving parents. 


\section{Table of Contents}

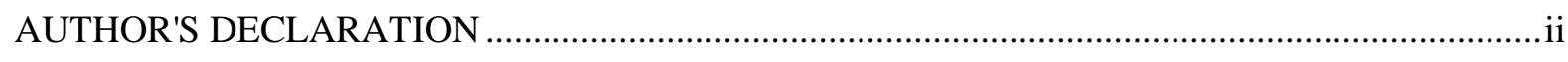

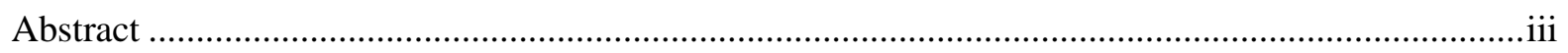

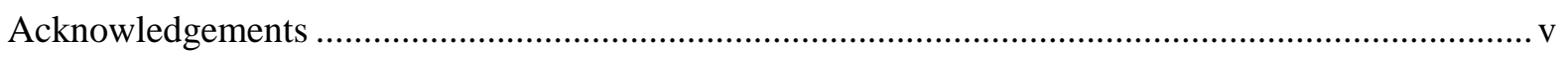

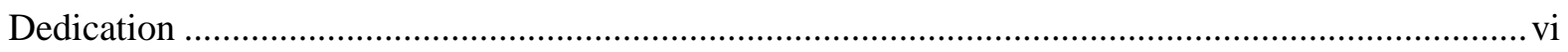

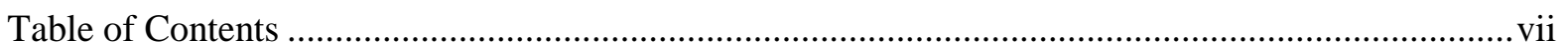

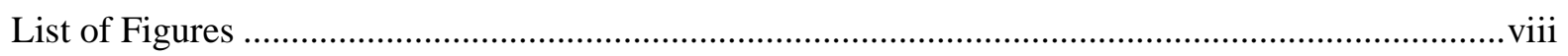

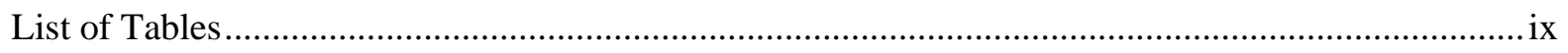

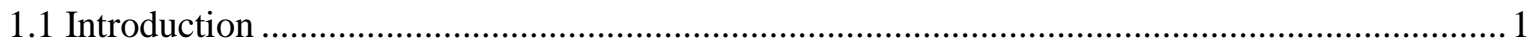

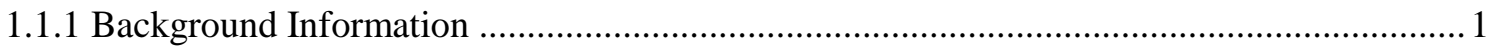

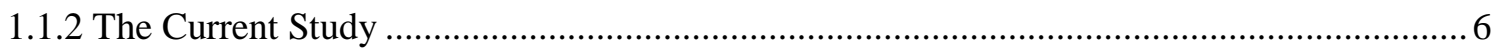

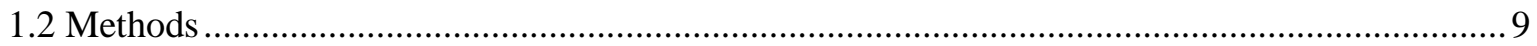

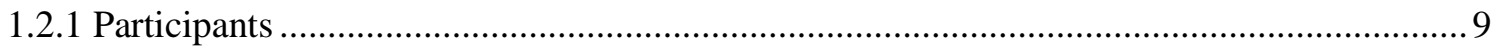

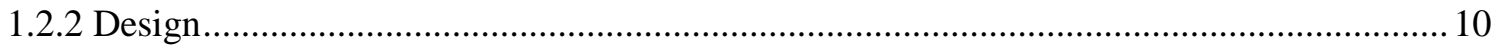

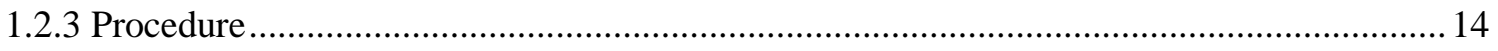

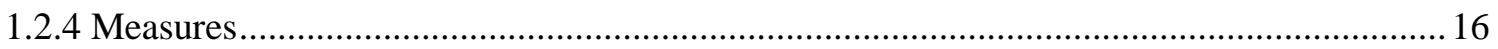

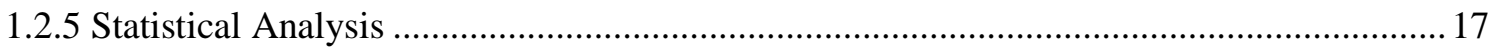

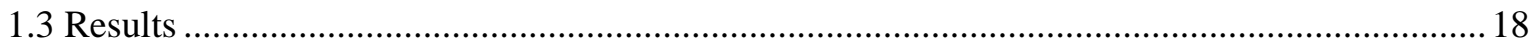

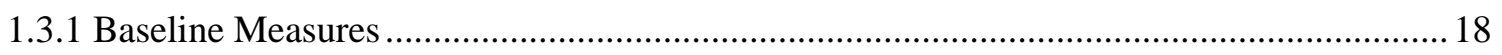

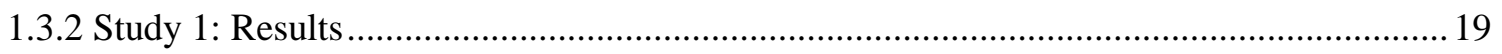

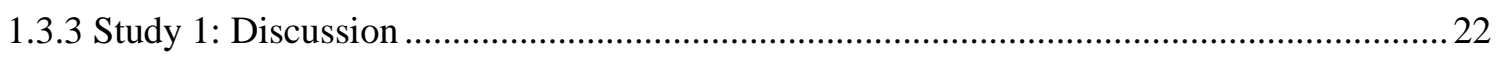

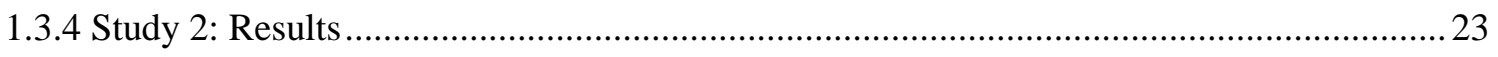

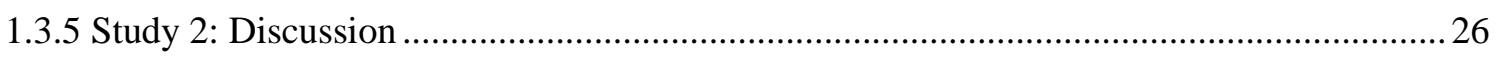

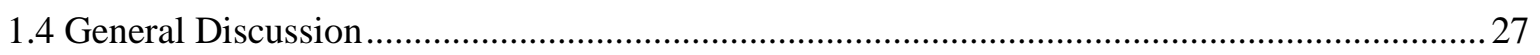

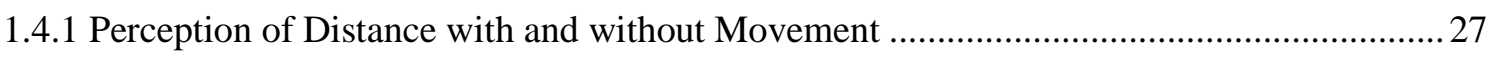

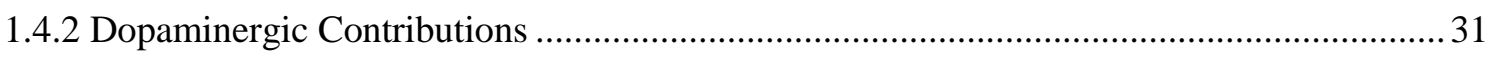

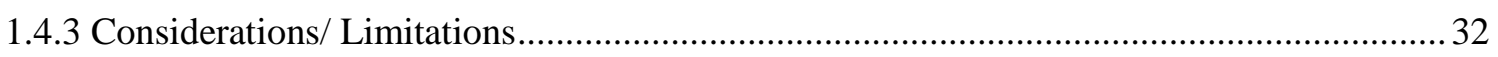

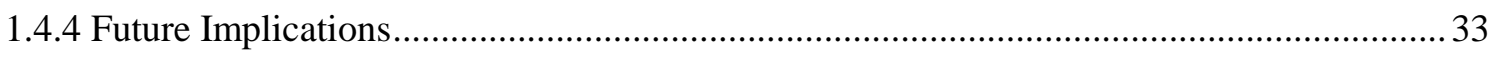

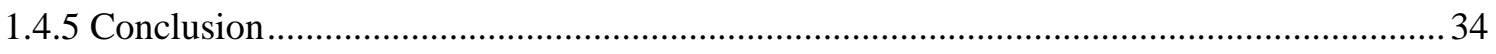

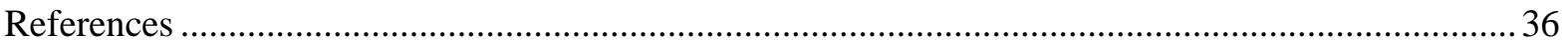




\section{List of Figures}

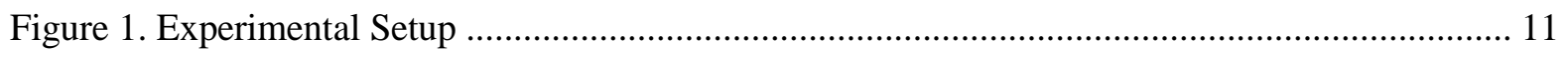

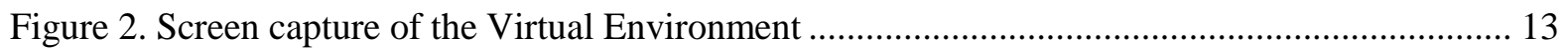

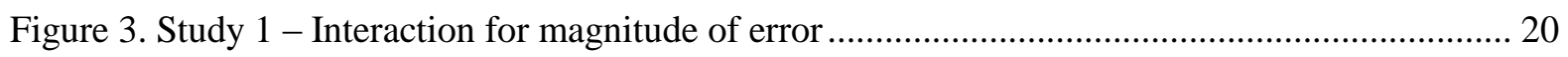

Figure 4. Study 1 - Main effects for direction of error ................................................................... 21

Figure 5. Study 1 - Dopaminergic influences on magnitude of error ................................................ 22

Figure 6. Study 2 - Interaction for magnitude of error ................................................................. 24

Figure 7. Study 2 - Dopaminergic influences on variability of error ............................................ 26 


\section{List of Tables}

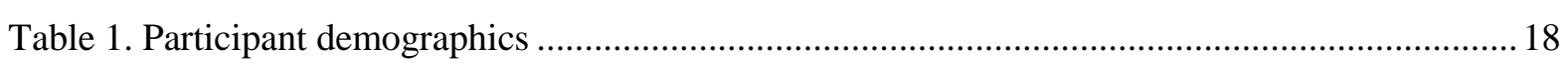





\subsection{Introduction}

\subsubsection{Background Information}

The basal ganglia (BG) are an important part of a complex neural network that processes and integrates various sensory inputs in order to produce and modulate motor outputs (Contreras-Vidal, 1999; Graziano \& Gross, 1993; Houk \& Wise, 1995; Nagy, Eordegh, Paroczy, Markus, \& Benedek, 2006). Boecker and colleagues termed the BG a "sensory analyzer" engaged in central somatosensory control, suggesting interconnections between the cortex, BG and thalamus that make up an indirect BG-sensory loop (Boecker et al., 1999b). In Parkinson's disease (PD) there is a degeneration of dopaminergic neurons in the BG, specifically in the substantia nigra pars compacta, which impairs the basal ganglia-cortical circuitry. This results primarily in motor symptoms, however implications of BG damage on sensory processes has remained uncertain. In the past decade, many researchers have examined sensory and perceptual capabilities in PD to try and explain common motor deficits such as hypometric movements, increased number of falls, poor balance and freezing of gait. Although a sensory-perceptual origin for motor deficits has been hypothesized (Almeida \& Lebold, 2010), the manner in which visual perception is integrated with proprioceptive processing in order to produce accurate movements through an environment remains unclear.

Previous research has shown that cognitive potentials evoked by visual stimuli were generated within the BG (Rektor et al., 2004). Johnson and colleagues (2004) investigated information processing in those with PD to see whether delayed processing might underlie movement slowness (Johnson et al., 2004). A perceptual speed paradigm was employed to examine the amount of time needed to perceive a stimulus in order to correctly identify its physical characteristics. Results from this study showed that individuals with PD required more time to identify stimulus properties while both 'on' and 'off' dopaminergic medication compared to healthy control participants, demonstrating that damage to the BG not only delays motor responses but importantly decreases 
perceptual speed for the processing of visual stimuli. Based on these combined results one might expect that those with PD may be unable to accurately process visual information, impairing their ability to properly perceive their environment.

Researchers have investigated whether those with PD are impaired in perceiving their visual environment. One study measured target pointing errors during a depth perception illusion task and found that PD participants had a significantly more errors during the pointing task compared to healthy control participants (Maschke, Gomez, Tuite, Pickett, \& Konczak, 2006). Similar results have also been identified when PD participants were asked to verbally estimate the depth of an environment. Some researchers have even suggested that individuals with PD demonstrate a perceptual asymmetry that distorts their visual representation of the environment. Large leftward deviations during a straight ahead pointing task (Wright, Gurfinkel, King, \& Horak, 2007) and compressed body-scaled judgments of aperture width (Lee, Harris, Atkinson, \& Fowler, 2001) have provided support for this hypothesis. Taken together it is important to consider how a visual impairment might affect subsequent movements through an environment.

Navigation through an environment is dependent on 1) accurate perception of objects in the environment, and 2) one's own body movement through an environment. It is possible that the inaccurate movements PD often display may reflect impairment to the visual perception system, such that individuals with PD misjudge the location or size of an object which is intended to be avoided (i.e., obstacles, doorways, etc.). Some individuals with PD find it exceptionally difficult to navigate through a doorway. A phenomenon called freezing of gait (FOG) often presents itself when those with PD approach an upcoming doorway. FOG appears as a transient episode in which the individual is unable to generate a step. Importantly, this phenomenon is not present in all individuals with PD (Bloem, Hausdorff, Visser, \& Giladi, 2004; Giladi et al., 2001). There have been many hypotheses that have tried to explain this phenomenon, including one which suggested that FOG may have 
perceptual origins (Almeida \& Lebold, 2010). However, very few studies have investigated this hypothesis, and those that have, have shown conflicting results. A recent study investigated FOG and perception by varying the width of a doorway (narrow, normal, and wide) through which participants were required to walk. Results showed that spatial-temporal aspects of gait worsened in the PD-FOG patients when approaching the narrow doorway, providing support for the hypothesis of an underlying perceptual mechanism that may interfere with online movement planning in PD (Almeida \& Lebold, 2010). Another study replicated this protocol (varying the widths of a doorway) but also had participants make a perceptual judgment of the doorway width after passing through the doorway. Here, results showed that similar to the previous study, gait worsened in PD when approaching the narrow doorway, however, body-scaled judgments of the doorway width did not differ from those of healthy control participants, and no effects of dopaminergic medication were found (Cowie, Limousin, Peters, \& Day, 2010). Therefore, these researchers did not find evidence of a perceptual judgment deficit in PD after they had walked through the doorway, yet FOG was still worse in the narrow width condition. Similarly, a different study asked participants to make perceptual height judgments of an obstacle in their environment. Those with PD did not differ from healthy control participants in their perceptual judgment accuracy (Martens \& Almeida, 2011). Taken together, there appears to be some evidence of a visual processing impairment of the environment in PD, however when tested in an ecologically valid setting (i.e., prior to crossing an obstacle, or after passing through a doorway), there does not seem to be clear evidence of a perceptual deficit within PD (even though FOG does appear to be modulated by manipulation of the physical environment). Therefore, a perceptual mechanism should be ruled out since it has only been tested in various forms of a static judgment task (i.e., after participants have walked through the doorway, or standing judgments of obstacle height). It is possible that those with PD are unable to process other sensory information 
about movement, such as optic flow (i.e., visual information about movement direction) or selfmotion which may lead to subsequent movement impairments.

There has been limited research investigating whether movement impairments in PD could be related to processing of visual information that is derived from forward motion. Evidence of a visual processing deficit resulting from impairment of the magnocellular system (i.e., an area in the brain that processes visual information about movement) has been hypothesized (Castelo-Branco et al., 2009; Silva et al., 2005), since peripheral vision is known to be altered in PD by an abnormality of dopaminergic amacrine cells (Bodis-Wollner \& Paulus, 1999). There has been some support for this hypothesis since studies have shown that those with PD have difficulty identifying the direction or speed of movement when solely relying on moving visual stimuli in their periphery (Mosimann et al., 2004; Trick, Kaskie, \& Steinman, 1994) . However, research investigating motion discrimination in PD did not find that the damaged magnocellular pathway was predictive of motion integration deficits (Castelo-Branco et al., 2009). It is possible that movement impairments are not the result of inaccurate processing of visual information during movement, however this has yet to be studied.

Accurate movements also depend on one's ability to track displacement through space using path integration (i.e., using sensed motion to update the current position and orientation of one's body relative to a starting position) (Durgin et al., 2005; Rieser, Pick, Ashmead, \& Garing, 1995). The somatosensory system is very important when moving through an environment since it stores proprioceptive information generated from the trunk and legs during locomotion, which is then integrated with vestibular information to compute current position (Barlow, 1964). Therefore, it is essential to consider the role of proprioception in motor production and control.

Proprioceptive deficits are well documented in PD (Klockgether, Borutta, Rapp, Spieker, \& Dichgans, 1995; Schneider, Diamond, \& Markham, 1987; Zia, Cody, \& O'Boyle, 2000), although similar to visual perception, their contribution to movement impairments remains unclear. Studies 
have shown that individuals with PD have abnormal processing of sensory information, specifically proprioceptive inputs (Klockgether et al., 1995; Rickards \& Cody, 1997; Schneider et al., 1987; Zia et al., 2000). Abnormal vibration-induced movement errors have been observed in PD during joint flexion-extension tasks(Rickards \& Cody, 1997; Schrader et al., 2008), and PET imaging studies of passive finger vibration have demonstrated a reduction in sensory evoked brain activations in the cortical (parietal and frontal) and subcortical (BG) areas of the brain (Boecker et al., 1999a). During limb position tasks, individuals with PD make more errors then healthy control participants when required to identify the occurrence and/or direction of passive movements (Schneider et al., 1987) as well as demonstrating greater errors in matching static limb position, detecting limb displacements, or using the lower limbs to estimate the size of an object (Martens \& Almeida, 2011; O'Suilleabhain, Bullard, \& Dewey, 2001; Zia et al., 2000). Similarly, tasks involving reaching and pointing to remembered targets have found that PD patients exhibit significantly large errors when locating the original target (Keijsers, Admiraal, Cools, Bloem, \& Gielen, 2005; Klockgether et al., 1995), especially when patients are unable to see their hand (Mongeon, Blanchet, \& Messier, 2009) or in the complete absence of visual information (Keijsers et al., 2005). Adamovich and colleagues (2001) took these findings one step further, and showed that reaching accuracy of PD deteriorated selectively when two sources of sensory information needed to be integrated with one another (i.e., visualproprioceptive integration), which presented uncertainty as to whether spatial errors of PD arise from deficits in proprioceptive processing or from difficulty in visual-proprioceptive integration (Adamovich, Berkinblit, Hening, Sage, \& Poizner, 2001). There is converging evidence that undoubtedly points to a proprioceptive deficit in the upper limbs, however, very few studies have investigated whether these movement impairments translate into the lower limbs. Self-motion is governed by proprioceptive feedback in the lower limbs which contribute to and modulate balance, locomotion and navigating through an environment since this is a much more continuous, repetitive 
and coordinated movement. Furthermore, one would expect that the lower limbs may be more dependent on proprioception since vision is less involved in guiding movements compared to the upper limbs. Therefore, movement impairments might be anticipated in PD as a consequence of poor proprioceptive processing. One study in particular investigated the influence of visual and proprioceptive information in locomotion and target accuracy in PD (Almeida et al., 2005). Results showed that when PD patients performed the task 'off' their medication, they moved to the target (in the dark) with less accuracy than healthy control participants and demonstrated the most difficulty when proprioception was the primary source of feedback. These findings support the hypothesis that locomotion may be heavily dependent on proprioception which is impaired in PD, thus influencing movement accuracy. Jacobs and Horak (2006) employed a similar paradigm measuring compensatory stepping behaviours in PD. They also found that individuals with PD made larger errors when stepping to a target and those with severe PD were particularly disadvantaged when they were prevented from seeing their legs (Jacobs \& Horak, 2006). Jacobs and Horak noted however, that even when participants were allowed full vision, step accuracy was still compromised. This suggested that in severe PD visual input could not fully compensate for a proprioceptive deficit. . However, it has yet to be determined whether self-motion deficits can be overcome by visual feedback during movement through a regular environment, especially since the majority of the previous work has focused on proprioceptive deficits and movement impairments in complete darkness. The current research will attempt to replicate real world situations where vision and proprioception are both used to move through an environment, to see whether it becomes clear which sensory system might be contributing to movement impairments experienced in PD.

\subsubsection{The Current Study}

The integration of many different sensory cues such as vision, proprioception, vestibular as well as a motor efference copy all contribute to accurate movements when navigating through an environment. 
It has been suggested that individuals with PD may have sensory perceptual deficits that could contribute to commonly seen movement impairments, however, it remains unknown which sensory cues are problematically processed during movement in PD. The overall goal of the current study was to examine sensory perceptual deficits across all sensory modalities in PD to gain a better understanding of the underlying causes of movement problems.

One method of examining sensory perceptual deficits is to use a distance estimation paradigm, and assess the accuracy of judgments made with different types of sensory information available. Thomson (1983) showed that it was possible to walk to a target accurately, with only a short visual presentation and no further subsequent visual input necessary. He noted that individuals must calibrate the visual representation of a target location with other sources of sensory information to accurately walk to the target. This study led to many studies that manipulated sensory conditions in young healthy individuals, in order to understand how different sensory systems contribute to accurate distance perception. Thus, a variety of approaches to estimate distance were used in the current study each to individually assess the accuracy and contribution of different sensory modalities. This protocol will provide insight into which "sensory calibration" may be impaired as a result of damage to the basal ganglia in those with PD. In addition, the current study will provide a further understanding of how sensory impairments contribute to movement deficits through an environment.

Many previous studies have used a similar paradigm to investigate multi-sensory contributions to distance estimation and path integration in healthy young adults (Bigel \& Ellard, 2000; Ellard \& Shaughnessy, 2003; Ellard \& Wagar, 2008; Lappe \& Frenz, 2009; Loomis, DaSilva, Philbeck, \& Fukusima, 1996; Redlick, Jenkin, \& Harris, 2001; Sun, Campos, Young, Chan, \& Ellard, 2004). Studies have shown that on average young adults are quite accurate in indicating location of targets under full sensory conditions (Loomis et al., 1996). Many researchers have also demonstrated 
that any sensory cue in isolation (i.e., visual, proprioceptive, optic flow) can provide sufficient information to be used to estimate distance accurately (Bigel \& Ellard, 2000; Bremmer \& Lappe, 1999; Ellard \& Shaughnessy, 2003; Harris, Jenkin, \& Zikovitz, 2000; Sun et al., 2004). These results demonstrate that young adults not only perceive the target location and their own self-motion correctly while walking, but they are also able to update their internal representation of a target based on their perceived self-motion. This allows them to execute the proper motor response that is directed toward the updated target location (Loomis et al., 1996). This was precisely what the current study aimed to investigate in $\mathrm{PD}$, in order to identity any disconnections within this path integration system that might lead to incorrect motor outputs.

Given that studies have shown that proprioception may be impaired with BG damage, it was hypothesized that individuals with PD would demonstrate inaccurate distance estimation in any conditions where participants were required to process proprioceptive information (i.e., walking to a remembered target position) compared to healthy age-matched control participants (HC). However, those with PD were expected to perform similar to age-matched control participants in static visual perception of distance, since previous studies investigating judgments of size (i.e., doorway width and obstacle height) did not find differences between those with PD and healthy control participants (Cowie et al., 2010; Martens \& Almeida, 2011). This would confirm that those with PD are able to correctly perceive the target location and build a spatial representation. Since locomotion also provides both visual motion cues (i.e., optic flow) and vestibular information in addition to proprioception, it was necessary to evaluate distance estimation accuracy with passive motion (removing proprioceptive input by passively pushing individuals in a wheelchair, or visually simulating forward movement in VR). Overall, in any condition where proprioception could be removed, it was predicted that no differences would be found between those with PD and healthy agematched control participants. 
To date, the role of dopaminergic medication on many symptoms of PD specifically nonmotor symptoms is unclear. There has been some evidence that dopaminergic medication makes proprioceptive processing worse in PD (O'Suilleabhain et al., 2001), however conflicting evidence has shown no difference between 'ON' and 'OFF' states (Almeida et al., 2005). In an effort to clarify the role of dopaminergic medication in sensory cue processing, the current study tested all PD participants both 'ON' and 'OFF' dopaminergic medication across sensory cue conditions. Therefore, the main objective of the present study was to evaluate (1) how distance estimation is influenced by static perception compared to perception during movement in $\mathrm{PD},(2)$ how visual motion processing contributes to distance estimation during movement, and (3) how dopaminergic medication contributes to these deficits.

\subsection{Methods}

\subsubsection{Participants}

This study involved 37 participants, 19 individuals with idiopathic PD (15 male, 4 female), and 18 healthy age-matched control participants ( 5 male, 13 female) recruited from the patient and healthy control participant database at the Sun Life Financial Movement Disorders Research and Rehabilitation Centre at Wilfrid Laurier University in Waterloo, Canada. To participate in the current study, all participants were required to speak and read English fluently, to not have seizures, vertigo, or motion sickness prior to the study (thus to reduce the risk of virtual reality simulator sickness), and also to have normal or corrected vision. Participants were excluded from the study if they had visual disturbances that would impair their distance acuity ( $>20 / 50)$, contrast sensitivity $(<18$ score on PeliRobson chart), gait impairments preventing individuals from walking 10 meters unassisted, dementia ( $<70$ score on Modified Mini Mental State Exam) or spatial working memory impairments $(<$ level 3 on Corsi block tapping task). All patients had been previously diagnosed with PD by a movement 
disorder neurologist. Patient files were carefully screened for co-morbid conditions (i.e. diabetes, history of stroke) prior to participation. (See Table 1 for participant demographics)

\subsubsection{Design}

\subsubsection{Apparatus}

Participants completed judgment trials in a clutter free large laboratory with free standing white walls at the edge of the room, on either side of the participant preventing additional visual cues in the environment (Figure 1). The participants stood at one end of a large laboratory room, and a $7 \mathrm{~m}$ white runner carpet was placed on the floor in front of them length-wise. This carpet was used to prevent the floor pattern from interfering or aiding individuals' judgments of distance. In addition, this carpet increased the contrast between the floor and the black box used as a target. Three infrared light emitting diodes (IRED) were fastened securely to the rear facing side of the black target box $(28.8 \mathrm{~cm} \times 20 \mathrm{~cm} \times 11.1 \mathrm{~cm})$. Two OPTOTRAK cameras were placed at the end of the room, 1 meter from the end of the carpet in order to capture the target's position. The target distance was measured at the beginning of every trial with a measuring tape and ranged from $1.4-6 \mathrm{~m}$ since previous literature has shown that prior vision can guide locomotion accurately (within $24 \mathrm{~cm}$ in young adults) up to 9m (Glasauer, Amorim, Vitte, \& Berthoz, 1994; Thomson, 1983; Loomis, Da Silva, Fujita, \& Fukusima, 1992).

A Snellen eye chart and Peli-Robson contrast sensitivity chart were used to measure distance acuity and contrast sensitivity, and a Corsi block apparatus was used to measure spatial working memory during the collection of baseline measures. 


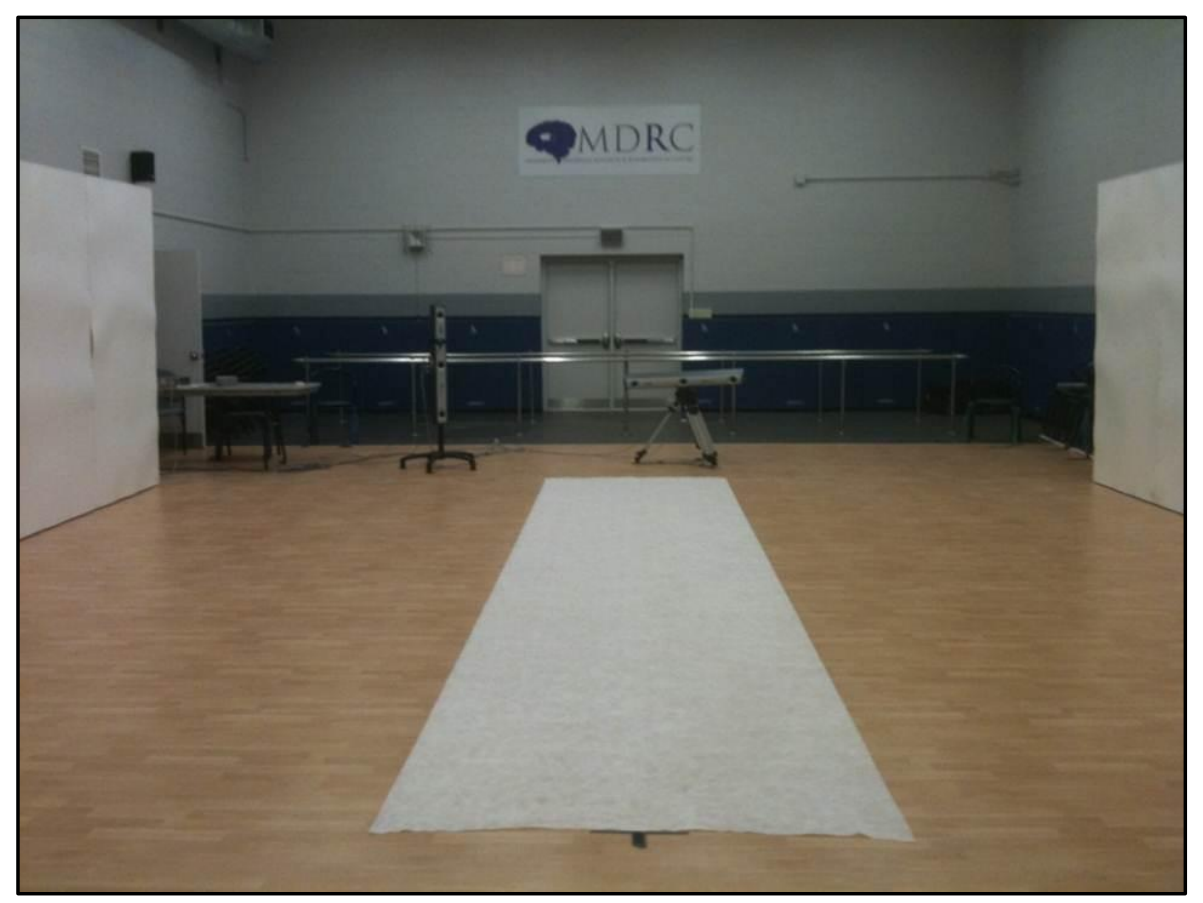

Figure 1. Experimental Setup

\subsubsection{Technical Information about Virtual Reality Setup}

The virtual environment used in this study was constructed using Google Sketchup modeling software and Worldviz virtual reality software, Vizard. The virtual environment was built to replicate the MDRC large laboratory, by creating an environment to scale of the real experimental setup to ensure a similar amount of visual motion and ultimately a similar experience as real world, since accuracy and precision of perceived distances is highly dependent on the properties of the surrounding environment (Lappin, Shelton, \& Rieser, 2006). The overall layout of the experimental setting was kept consistent by matching the texture and colour of the floor, walls and ceiling, having free standing walls to make distance cues limited (matched in the real experimental setup), as well as matching the lighting as well as possible (see Figure 2).

The testing environment was delivered in stereo mode to a V6 head-mounted display (HMD) that featured a 60 -degree field-of-view with a 640 x 480 dpi resolution. The HMD also featured a 
thick light-blocking cover that was pressed firmly to the participants' face, which prevented participants from seeing the real-world environment around them, and allowed them to focus only on the virtual environment. The viewpoint was controlled by an InterSense InertiaCube 2 head-tracking device which was attached to the HMD, allowing the viewpoint to update in real-time with physical head-movements in the medial-lateral and superior-inferior directions (i.e., if the participant turned their head to the right, the viewpoint would also look to the right in the virtual environment). Since participants were seated during this task (to avoid fatigue, disorientation and dizziness) participants' eye heights were inputted to allow the environment to appear as if participants were standing in a similar location as in all other real-world conditions. In addition, participants' individual regular walking elocity (as measured in the pre-test) was used to determine the pace of passive movement through the environment, to ensure their experience was as similar and immersive as possible. Movement through the virtual environment was achieved passively when the experimenter pressed a start button, passive simulated forward movement was initiated and the participants were to indicate with a mouse click, the time at which that target's position was reached, in similar fashion to the protocol used by Redlick et al., (Redlick et al., 2001). The direction of movement corresponded to the orientation of their head in the physical world. Participants were instructed to orient their body to the direction of the back wall looking straight ahead in order to reduce the mismatch between their physical bodies and virtual bodies. 


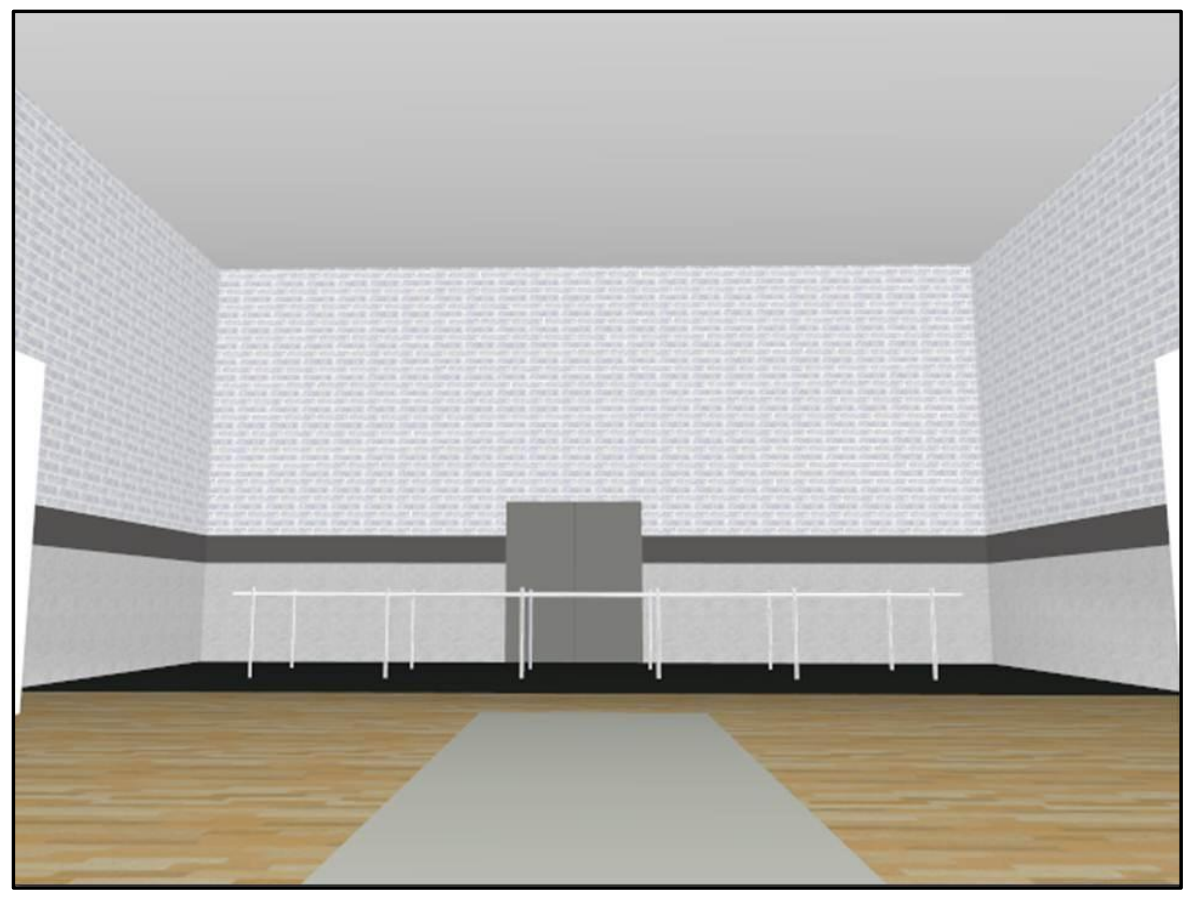

Figure 2. Screen capture of the virtual environment

\subsubsection{Data Collection}

Two separate experiments, each addressing a distinct research question, were carried out and collected simultaneously. The first study (i.e., Study 1) addressed the question of whether deficits during distance estimation exist as a result of visual perception deficits (no motion) or proprioceptive deficits (motion). The second study (i.e., Study 2) investigated perceptual judgment accuracy during motion without physical movement (i.e., only visual motion with and without vestibular input) to identify whether visual motion information contributes to distance estimation errors during movement in PD. All participants completed 4 randomized blocked conditions assessing perceptual estimates of distance. The 4 conditions included: 1) no motion, 2) motion, 3) visual motion (wheelchair), 4) visual motion (VR). The order of all 4 conditions completed was counterbalanced across all participants. During each condition there were 12 randomized trials with random distances assessed. The distances used were equated across conditions. Procedures and measures were identical between all 
conditions. The only difference between conditions was the way the participant expressed their distance estimate (i.e., with a laser, walking, passively moving, or clicking a mouse). Upon arrival at the Centre all participants completed informed consent followed by 3 baseline measures (distance acuity, contrast sensitivity, velocity). Participants then completed a total of 48 experimental trials (4 conditions x 12 trials), followed by 4 more baseline measures (3MS, Corsi block tapping test, 6 motor imagery trials and MIQ - revised version). All PD participants were assessed by a certified clinician using the Motor Section of the Unified Parkinson's Disease Rating Scale (UPDRS-III) to assess motor symptom severity at the time of testing.

In order to assess dopaminergic contribution to sensory perception, participants with PD completed the full protocol twice, once OFF their dopaminergic medication (after a minimum of 12 hours withdrawal) and again ON their optimal dosage of medication (approximately one hour after taking their regular dosage of medication). There was approximately 1 week between testing sessions between $\mathrm{ON}$ and $\mathrm{OFF}$ testing, and half of the PD participants were tested ON their regular dopaminergic medication first, and the other half were tested OFF medication first to avoid learning or practice effects.

\subsubsection{Procedure}

\subsubsection{Study 1: Perception with and without motion}

The two conditions in study 1 examined perception of distance with and without movement. During perception without movement (condition 1) participants were instructed to close their eyes while a target was placed at a measured distance from the participant. Once the target was in position, the participant was instructed to open their eyes and look straight ahead at the target and inform the researcher when they were ready for the target to be removed (i.e., they are confident they know the position of the target). Once the participant indicated they were ready, they were asked to close their 
eyes, the experimenter removed the target, and then the participant was told to open their eyes and point with a laser pointer to the position formerly occupied by the target. Once the participant pointed to the estimated position, the experimenter placed the target at their estimated distance and confirmed that this was the position of the target. Participants were allowed as much time as they needed to view the target prior to it being removed as well as unlimited amount of time to estimate the target's position. This was an effort to avoid any deficits related to inspection time or time to process the position of the target. This procedure was repeated for all 12 trials. During perception with motion (condition 2), participants completed exactly the same protocol as described above, but instead of pointing with a laser to give their estimate of distance, they were instructed to walk to the position of the remembered target, and stop when their feet were at that position. A spotter would follow closely behind the participant, but off to the side in order to ensure the participant's safety. Once the participant had stopped, the experimenter would place the target on the ground at their feet and confirm with the participant the position of their estimate. This procedure was repeated for all 12 trials.

\subsubsection{Study 2: Perception with visual motion only}

The two conditions in study 2 examined perception of distance during motion, however with no proprioceptive information, only visual optic flow and vestibular information. Two conditions assessed judgment accuracy: perception with visual motion +vestibular information (wheelchair) and perception with visual motion only (VR). Of these two conditions, one was done in a real world setting with passive movement induced by pushing participants in a wheelchair, however the other condition was performed in a virtual setting that replicated the experimental testing space. These two conditions aimed to probe for deficits in processing visual motion among participants with $\mathrm{PD}$, and to compare distance estimation in real and virtual settings. The procedure in these two conditions was the same as in Study 1, except that the method of distance estimation was varied. During the 
wheelchair condition, participants were shown the target, and then participants were pushed in a wheelchair down the carpet, until the participant indicated that they had reached the position of the remembered target. The target was then placed at their feet where participants confirmed their estimate to be. During the virtual reality condition, participants were seated, wearing the HMD, and viewed the virtual environment in the HMD. Participants were still shown the target in the virtual environment (VE), and then once the participant was ready, the participant was instructed to close their eyes, the target was removed, the participant was told to open their eyes, and to indicate when they were ready to move forward. The experimenter then pressed a button that began the trial, simulating forward movement through the VE as though the participant was moving towards the end of the carpet. The participant was instructed to indicate with a mouse click when they had reached the position at which they remembered the target, and then the trial ended.

\subsubsection{Measures}

The 3 IREDs were used to derive the actual position of the target, measured by the researcher and shown to the participant, and then the participants estimated position of the target. Distance estimation errors were calculated using three different error measures: absolute, constant and variable

error. Absolute error was used to indicate the magnitude of each estimate error and was calculated by subtracting the actual distance from the estimated distance and then taking the absolute value (i.e., absolute error $=\mid$ estimate-actual $\mid)$. Constant error was used to indicate the direction of the estimate error, for example, whether the participants underestimated or overestimated the distance, by subtracting the actual distance from the estimated distance (i.e., constant error $=$ estimate - actual). Variable error was used to indicate the variability of the participants' distance estimations. It was measured by calculating the standard deviation of the constant error across all 12 trials. During the virtual reality condition, OPTOTRAK was not used since the box was presented in the virtual environment; the estimate was calculated automatically from the VR script that presented the trial. 
Baseline measures included assessments of distance acuity, contrast sensitivity, spatial working memory, velocity, 3MS, UPDRS (PD only) and motor planning. Motor planning was assessed using a similar protocol as (Bakker, de Lange, Stevens, Toni, \& Bloem, 2007; Snijders et al., 2011) by having participants walk to a mark on the floor (either $2,4.5,6 \mathrm{~m}$ away) and return to their start position. The experimenter measured the time it took for the participant to complete this walking task. After this was complete, the participant was then asked to imagine walking to the mark they had just walked to and returning to the start position. The participant was instructed to indicate when they had returned. The experimenter then measured the time it took for the participant to imagine completing this trial. The difference between the actual time to complete the walking task and the imagined time to complete the walking task was compared.

\subsubsection{Statistical Analysis}

There were two independent groups involved in this study: individuals with Parkinson's disease (PD) and healthy control participants (HC). The primary dependent variable was the magnitude of error in participants estimated judgments (i.e., absolute error). Other dependent variables included direction of error (i.e., constant error) and the variability of error (i.e., variable error).

Independent t-tests were conducted to determine whether there were significant differences in age, dementia, contrast sensitivity and motor planning between those with PD and healthy control participants. A nonparametric Mann-Whitney U test compared distance acuity and spatial working memory between PD and healthy control participants since this was ordinal data.

Study 1 and Study 2 were analyzed separately using two mixed ANOVAs and Tukey's post hoc tests to determine whether there were significant differences between PD-OFF and healthy control participants. Additionally, two repeated measures ANOVAs were conducted to determine whether there were significant differences within PD-OFF and PD-ON in order to understand the contribution of the dopaminergic system in Study 1 and Study 2. 
Distance and trial effects were investigated in a separate analysis (i.e., mixed ANOVA) for each condition, since the trials in each condition had random distances. Three short, medium and far distances were selected and categorized to compare whether distance impacted the participants judgment error in each condition. Each distance category ranged $30-70 \mathrm{~cm}$ and were arranged in the order that the trials were completed. This analysis investigated whether there was any interaction between group and distance.

\subsection{Results}

\subsubsection{Baseline Measures}

Independent t-tests confirmed that no significant differences existed between individuals with PD and healthy control participants for age $(\mathrm{t}(35)=0.74, \mathrm{p}=0.46)$, dementia $(\mathrm{t}(35)=1.32, \mathrm{p}=0.2)$, and motor planning ( $\mathrm{t}(35)=0.14, \mathrm{p}=0.89)$. Nonparametric tests confirmed that there were no significant differences in spatial working memory (Mann-Whitney $U=1.62 .5, \mathrm{p}=0.79$ ), and distance acuity (Mann-Whitney $U=152, \mathrm{p}=0.56$ ) between PD and healthy control participants. Individuals with PD did however score significantly lower on the contrast sensitivity test $(\mathrm{t}(35)=2.53, \mathrm{p}<0.05)$ compared to healthy control participants. (See Table 1).

Table 1. Participant demographics

\begin{tabular}{|lllllllll|} 
& Age & UPDRS- & 3MS & $\begin{array}{l}\text { Distance } \\
\text { Acuity }\end{array}$ & $\begin{array}{l}\text { Contrast } \\
\text { Sensitivity }\end{array}$ & Corsi & Motor & MIQ \\
& & III & & Imagery & \\
\hline $\begin{array}{l}\text { PD-OFF } \\
(n=19)\end{array}$ & 71 & 32 & 96 & $20 / 30$ & 30 & 4.3 & 1.9 & 75 \\
\hline $\begin{array}{l}\text { PD-ON } \\
(n=19)\end{array}$ & $-\cdots$ & 23 & 95 & $20 / 30$ & 29 & 4.1 & 1.8 & 78 \\
\hline $\begin{array}{l}\text { HC } \\
(n=18)\end{array}$ & & & & & & & & \\
\hline
\end{tabular}


Note: UPDRS-III: Unified Parkinson's Disease Rating Scale- Motor Section, 3MS: Modified Mini Mental State Exam, Corsi: Corsi block tapping task, MIQ: Motor Imagery Questionnaire.

\subsubsection{Study 1: Results}

\subsubsection{Magnitude of Error}

Main effects for group $(\mathrm{F}(1,35)=8.27, \mathrm{p}<0.01)$ and condition $(\mathrm{F}(1,35)=10.48, \mathrm{p}<0.005)$ were found during perceptual distance estimations. More importantly, a condition by group interaction (F $(1,35)=4.21, \mathrm{p}<0.05)$ was found. PD judgment errors did not differ significantly from healthy control participants' during static judgments (no motion condition) but PD patients had significantly larger errors than healthy control participants when their judgments were given by walking to the target's position (motion condition). Post hoc tests also revealed that although HC participants did not differ in judgment errors in the static versus the motion condition, Those with PD demonstrated greater error in the judgments requiring movement (motion condition) compared to their static judgments (no motion condition). The significant interaction between condition and group is shown in Figure 3.

Within the static judgments, there was a significant distance by group interaction $(F(2,70)=$ $5.58, \mathrm{p}<0.01)$ which demonstrated that PD-OFF had significantly greater amounts of error when estimating the closest distances compared to healthy control participants. 


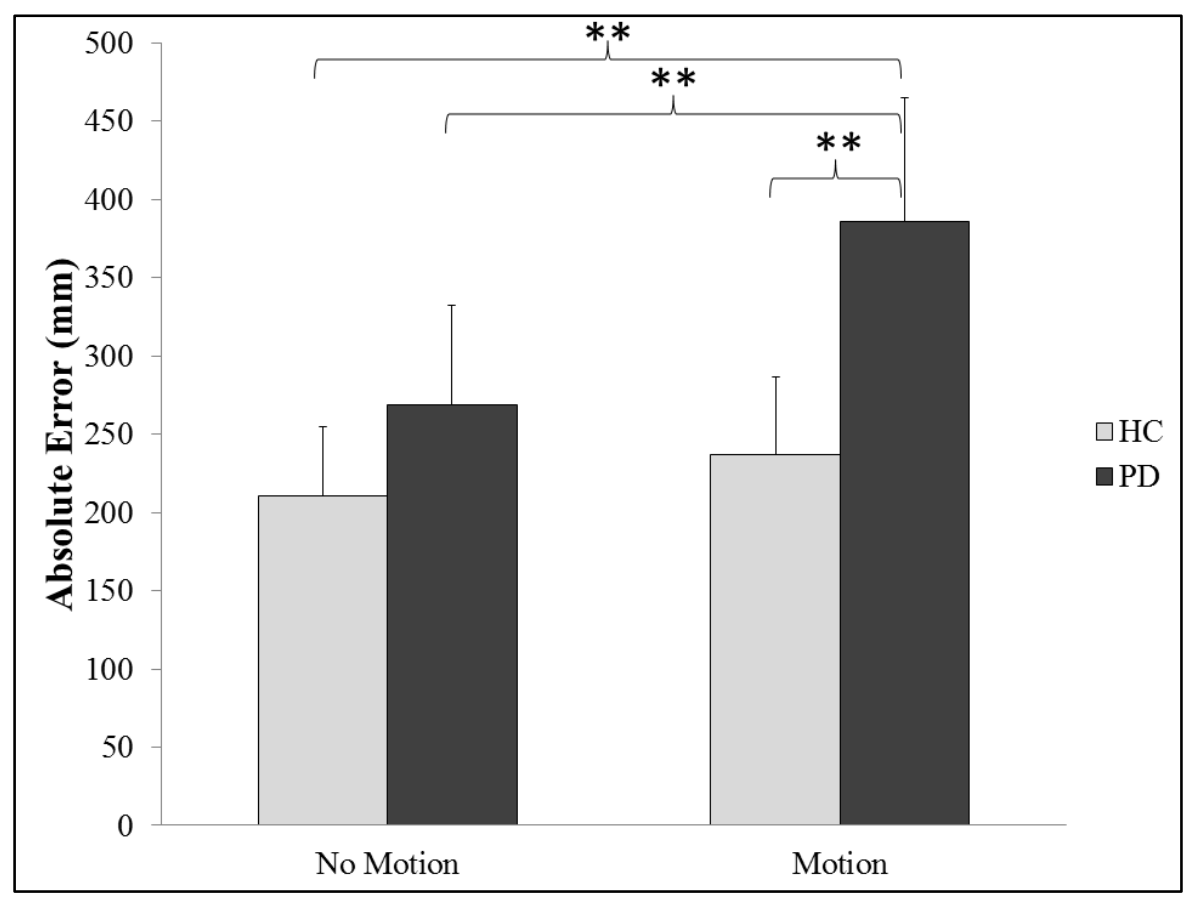

Figure 3. Study 1 - Interaction for the magnitude of error. Error bars represent 1 S.E. Mean. $* *$ indicates $\mathrm{p}<0.005$.

\subsubsection{Direction of Error}

Main effects for group $(\mathrm{F}(1,35)=8.77, \mathrm{p}<0.01)$ and condition $(\mathrm{F}(1,35)=35.38, \mathrm{p}<0.0001)$ were found during perceptual distance estimations, demonstrating that overall PD underestimated the target distance more than healthy control participants, however both groups underestimated target distance more during their static judgments compared to judgments made after moving to the target position. Planned comparisons between groups within conditions demonstrated that individuals with PD underestimated significantly more than healthy control participants only during their judgments with movement (motion condition).

There was no significant distance by group interactions found within either condition. 


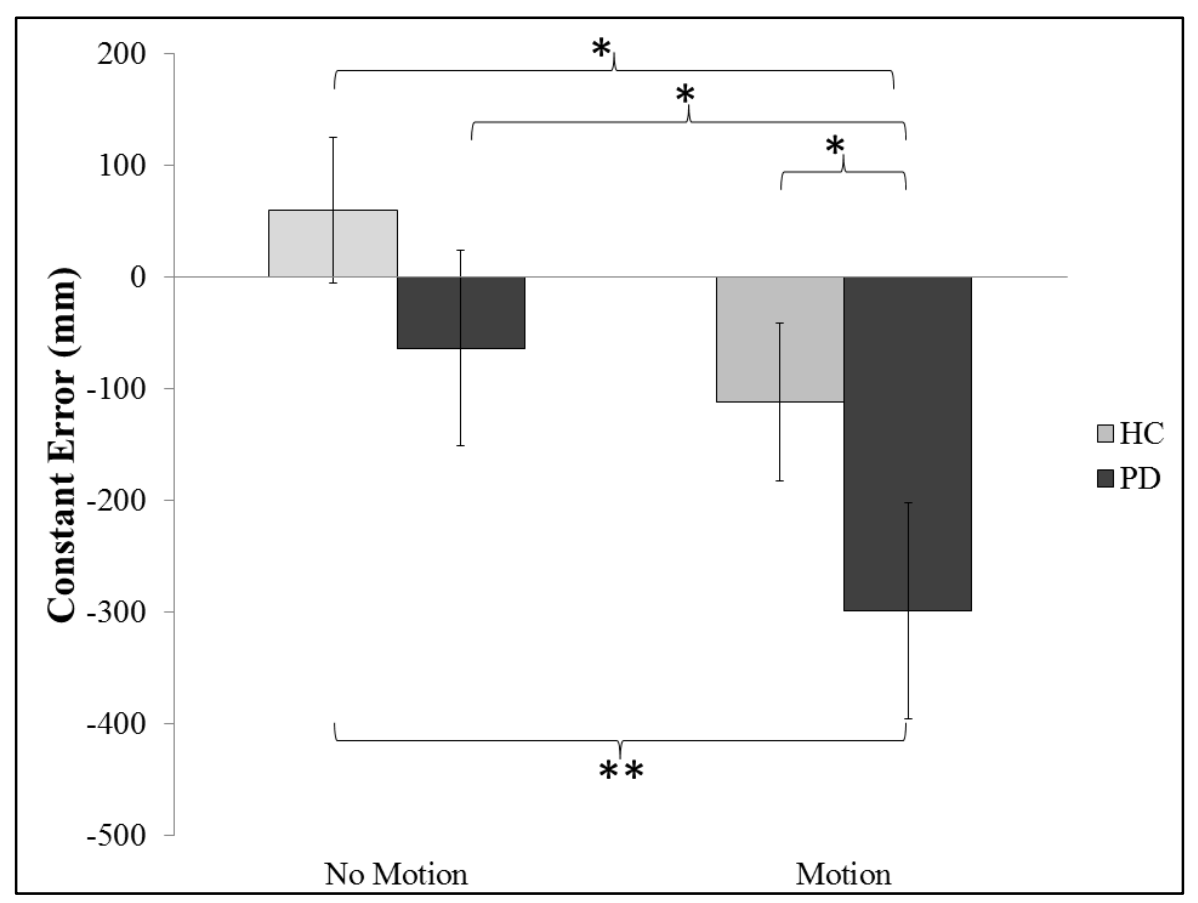

Figure 4.Study 1 - Main effects for direction of error. Error bars represent 1 S.E. Mean. *indicates $\mathrm{p}<0.05 ; * * \mathrm{p}<0.005$.

\subsubsection{Variability of Error}

A significant main effect for group $(F(1,35)=5.93, \mathrm{p}<0.05)$ revealed that $\mathrm{PD}$ had greater amounts of variability in their judgments during both conditions compared to healthy control participants.

\subsubsection{Dopaminergic Contributions to Error}

When comparing within-subject PD performance both OFF and ON dopaminergic medication, there were no significant main effects of medication in absolute, constant or variable error. There was however a main effect of condition in absolute error $(F(1,18)=16.88, p<0.001)$, and a main effect of condition in constant error $(\mathrm{F}(1,18)=28.64$, $\mathrm{p}<0.0001)$ demonstrating that PD participants had significantly greater amounts of error during judgments with movement compared their static judgments, and underestimated the target distance more during judgments with movement compared to their static judgments. Tukey's post hoc test revealed that there was only a significant difference 
between conditions when participants with PD were tested OFF their regular dopaminergic medication.

There was no significant distance by medication interactions found in either condition.

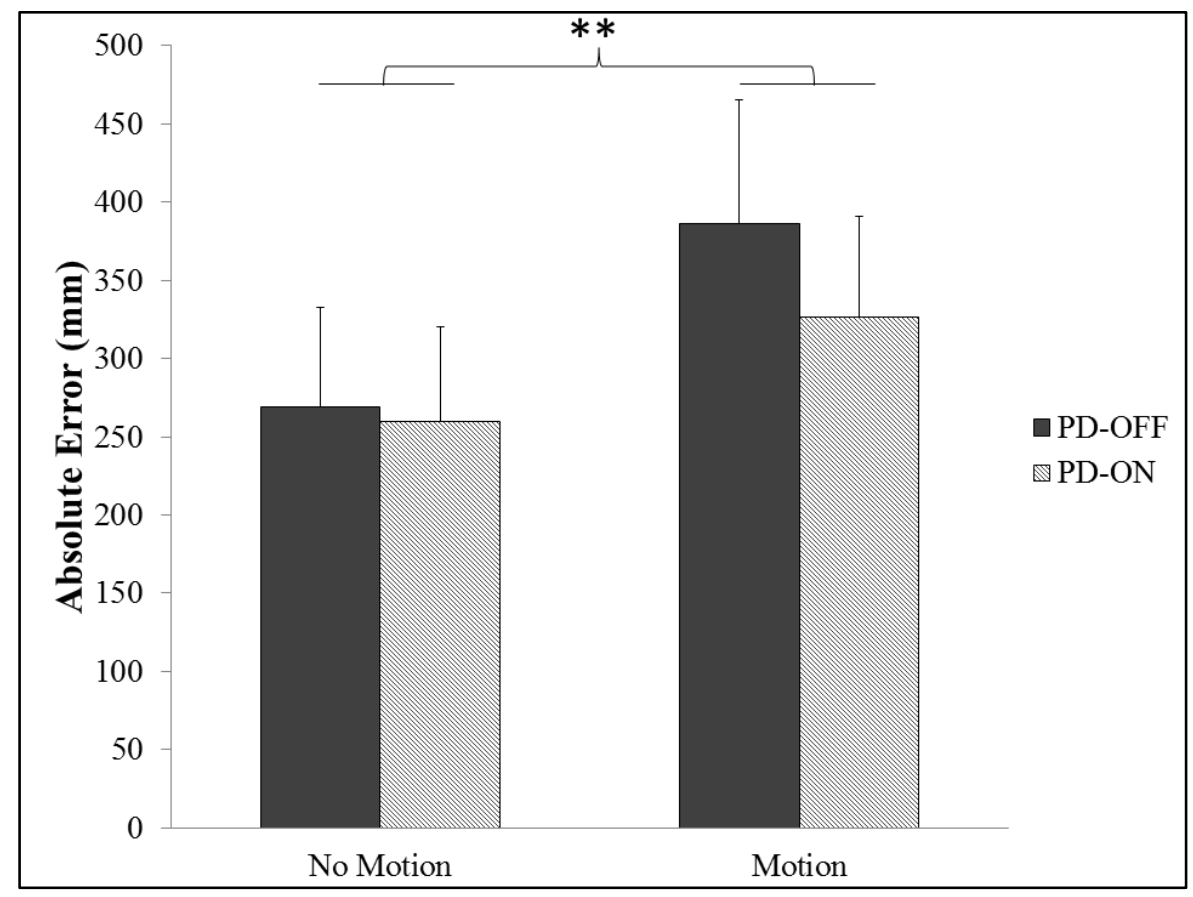

Figure 5. Study 1 - Dopaminergic influence on magnitude of error. Error bars represent 1 S.E. Mean. ** indicates $\mathrm{p}<0.005$.

\subsubsection{Study 1: Discussion}

The main findings from Study 1 demonstrates that individuals with PD have greater amounts of error in their judgments and underestimate the target position more than healthy control participants, specifically when they are required to move to their estimated position. However, individuals with PD did not differ from healthy control participants when required to stand and point to estimate the target's position. These results were able to fully address the original objectives of Study 1 and suggest that individuals with PD may have a self-motion perception deficit that leads to their inaccurate judgments of distance during movement and may also contribute to other movement impairments seen in PD. However, the current study did not find any evidence of a static perceptual 
deficit in PD, since those with PD did not differ from healthy control participants during their static perceptual judgment. Although dopaminergic replacement therapy did not significantly improve judgment accuracy in either perceptual task (with and without motion) in PD, PD-ON did not show significant differences in accuracy between the two conditions (as it was in the OFF state).

Therefore, it remains unclear how dopaminergic medication contributes to self-motion deficits in PD.

\subsubsection{Study 2: Results}

\subsubsection{Magnitude of Error}

A main effect for condition $(\mathrm{F}(1,35)=346.13$, $\mathrm{p}<0.0001)$ was found indicating that all participants demonstrated significantly greater amounts of error during the virtual reality condition compared to the real world wheelchair condition. Interestingly, a significant interaction between condition and group $(\mathrm{F}(1,35)=4.29, \mathrm{p}<0.05)$ showed that the healthy control participants had greater amounts of error during the VR condition compared to individuals with PD, whereas PD had slightly greater amounts error during the wheelchair condition. Tukey's post hoc test reveals a near significant difference $(\mathrm{p}=0.09)$ demonstrating that those with PD had less error than healthy control participants when estimating distance in VR (Figure 6). Within the VR condition, there was a significant distance by group interaction $(\mathrm{F}(2,70)=3.85, \mathrm{p}<0.05)$ which demonstrated that healthy control participants had significantly greater amounts of error when they estimated the furthest distances compared to PDOFF.

There was no significant distance by group interaction in the perception with visual motion (wheelchair) condition. 


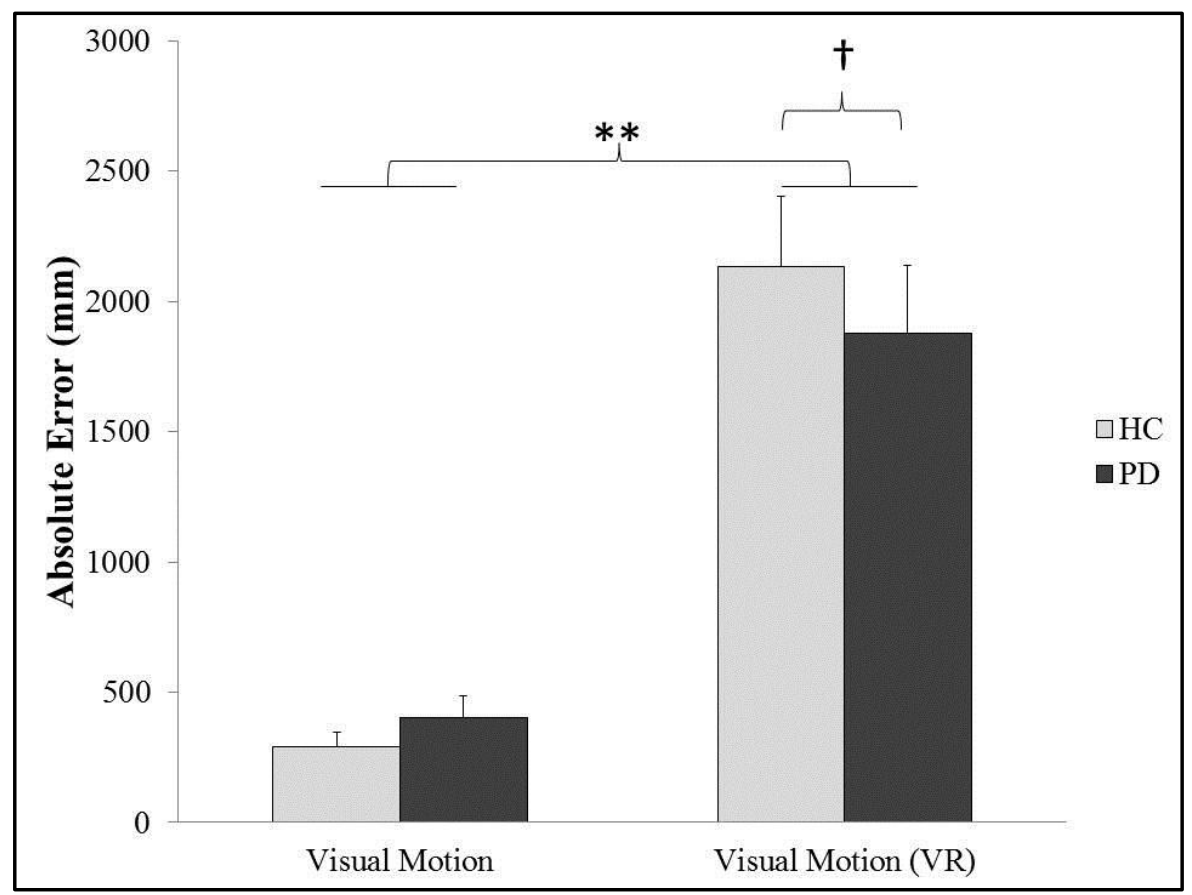

Figure 6. Study 2 - Interaction for magnitude of error. Error bars represent 1 S.E. Mean. $* *$ indicates $\mathrm{p}<0.005 ; \nmid$ indicates $\mathrm{p}<0.09$.

\subsubsection{Direction of Error}

Similar to the absolute error, the constant error showed a main effect for condition $(F(1,35)=164.63$, $\mathrm{p}<0.0001)$ demonstrating that all participants underestimated the distance of the target more during the VR condition compared to the wheelchair condition completed in the real world. A significant interaction between condition and group $(\mathrm{F}(1,35)=7.24, \mathrm{p}<0.05)$ showed that the healthy control participants underestimated the target distance more during the VR condition than individuals with $\mathrm{PD}$, whereas in the wheelchair condition those with PD underestimated the target location more than healthy control participants. Post hoc analysis confirms that healthy control participants significantly underestimated the target position in VR compared to those with PD $(\mathrm{p}<0.05)$.

There were no significant distance by group interactions found within either condition. 


\subsubsection{Variability of Error}

A main effect for condition $(\mathrm{F}(1,35)=840.07, \mathrm{p}<0.0001)$ showed that all participants had significantly more variability in their distance estimates during the VR condition.

\subsubsection{Dopaminergic Contributions to Error}

Similar to the findings of Study 1, when comparing PD performance both OFF and ON dopaminergic medication, there were no significant main effects of medication on absolute or constant error.

However, there was a significant main effect of medication on variable error $(\mathrm{F}(1,18)=6.21, \mathrm{p}<0.05)$ demonstrating that individuals with PD became significantly more variable in their error judgments when they were in the ON state compared to when they were in the OFF state (Figure 7). There was also a main effect of condition on absolute error $(\mathrm{F}(1,18)=224.76, \mathrm{p}<0.0001)$, constant error $(\mathrm{F}(1$, $18)=59.04, \mathrm{p}<0.0001)$, and variable error $(\mathrm{F}(1,18)=437.23, \mathrm{p}<0.0001)$ demonstrating that PD participants significantly underestimated the target distance with greater amounts of error and variability during perception in VR compared to the wheelchair condition regardless of their dopaminergic state.

There were no significant distance by medication interactions found in either condition. 


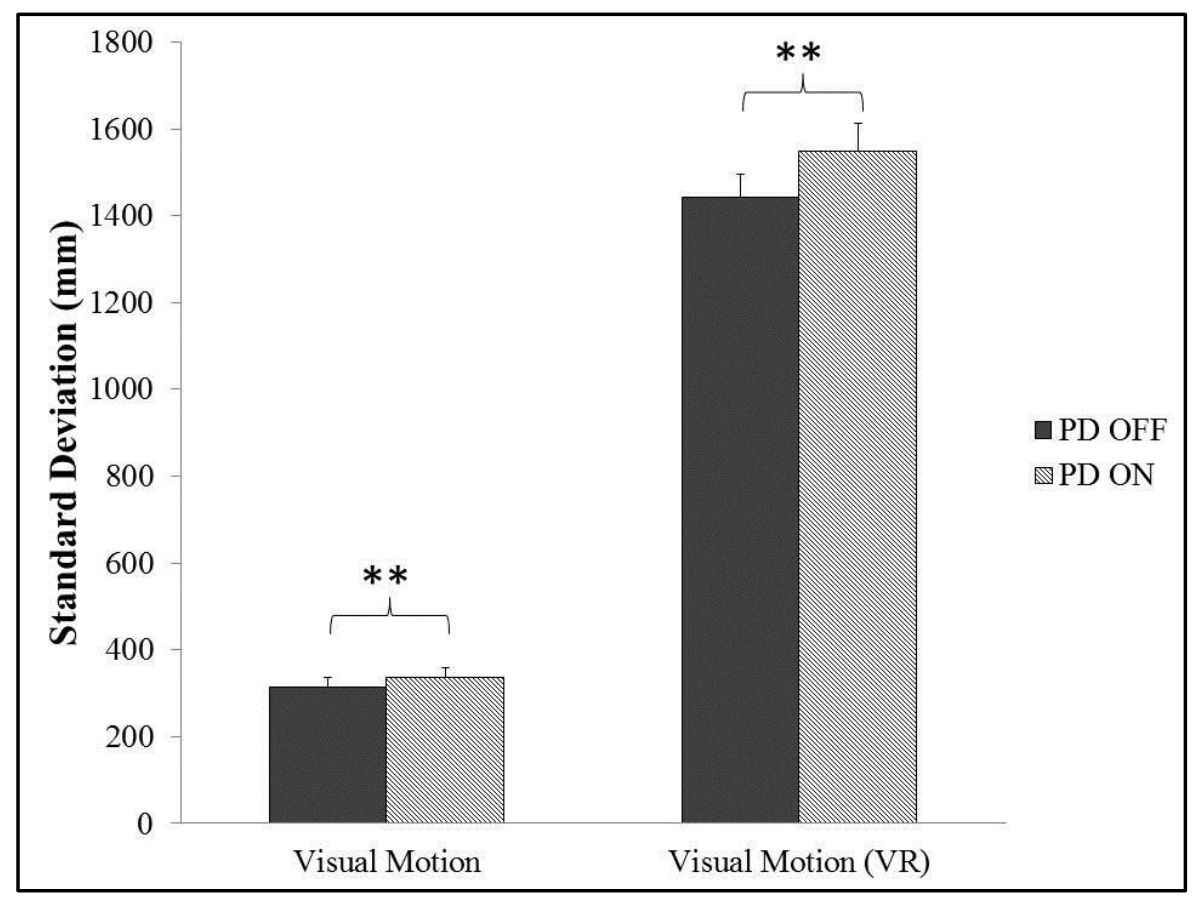

Figure 7. Study 2 - Dopaminergic influence on the variability of error. Error bars represent 1 S.E. Mean. $* *$ indicate $\mathrm{p}<0.005$.

\subsubsection{Study 2: Discussion}

The main findings from Study 2 demonstrates that individuals with PD do not display a deficit in processing visual information about motion (i.e., optic flow) to estimate distance, since their performance was similar to that of the healthy control participants. These results were able to fully address the main objective of Study 2, and confirm that self-motion deficits demonstrated in those with PD cannot be explained by impairments in processing visual motion information. Interestingly, those with PD demonstrated less error than healthy control participants when estimating distance in VR. This may illustrate the high level of dependence those with PD have on vision, since they may have been more immersed in the VR condition. Some researchers have suggested that those with PD are heavily dependent on vision in order to compensate for an underlying proprioceptive deficit, which might contribute to self-motion perception deficits seen in PD. 


\subsection{General Discussion}

The current study was able to address each of the main objectives by carefully considering a number of perceptual conditions (i.e., perception without motion, with motion, and with passive motion) that provided different types of sensory information, in order to answer whether a sensory-perceptual deficit exists in PD. The comparison of PD to healthy age-matched control participants provided a foundation to explore whether sensory or perceptual issues may be related to basal ganglia dysfunction in PD, since groups were matched for age, distance acuity, contrast sensitivity, spatial working memory, and motor imagery abilities. It was hypothesized that those with PD would demonstrate distance estimation errors during trials where participants were required to walk to the position of the target to give their distance estimate. This hypothesis was based on previous research suggesting that an underlying proprioceptive deficit or visual proprioceptive integration may be responsible for movement impairments commonly seen in PD (Almeida et al., 2005). Utilizing the different sensory conditions for estimating distance, impairments in processing visual information about distance, as well as processing visual motion information about moving through an environment were ruled out, since differences between those with PD and healthy control participants were not found. However, a deficit in perception with motion was identified and may suggest impairment in processing or integrating self-motion information.

\subsubsection{Perception of Distance with and without Movement}

The current study compared static (no motion) and dynamic (motion) distance estimations between PD and HC participants. There were no differences found between PD and HC during static distance estimation, demonstrating that individuals with PD were able to accurately perceive the target position and make an accurate spatial representation of the target location similar to HC. The most interesting finding however was that when PD participants were required to walk to the position of 
the target, they demonstrated significantly greater errors in their estimates of distance. They also underestimated the target location significantly more than healthy age-matched control participants. This suggests that a deficit exist in PD during self-motion, whereby PD patients are unable to accurately represent or update their own self-motion. These findings support the results found in previous studies investigating sensorimotor deficits in PD (Adamovich et al., 2001; Almeida et al., 2005), but importantly highlight that self-motion deficits are present even with vision of the environment. There are a number of potential explanations for the greater movement errors that were seen during the walking condition. First, proprioceptive processing of the upper limbs has been found to be impaired in PD (Keijsers et al., 2005; Klockgether et al., 1995; Rickards \& Cody, 1997; Zia et al., 2000). When investigating PD movements (i.e., pointing or walking) in complete darkness, thus relying solely on proprioceptive information, previous studies have found reliable error in PD similar to what was seen in the current study and have concluded that these errors were a result of proprioceptive deficits (Almeida et al., 2005; Jacobs \& Horak, 2006; Keijsers et al., 2005; Mongeon et al., 2009). A second possibility may be that, as has been demonstrated in previous research (Adamovich et al., 2001), visual-proprioceptive integration is impaired in PD. The current study had individuals walk to the position of the target with vision available throughout the entire trial. Therefore, it is not possible to dissociate between these two possible explanations since a manipulation of vision was not carried out. However, it is worth noting that even with full vision those with PD demonstrated significantly greater errors in their judgments, suggesting that vision could not fully compensate for a proprioceptive deficit. This provides strong ecological validity and evidence that movement through a daily environment may be impaired because of faulty proprioceptive processing and/or integration. This also supports previous findings that information gathered during locomotion contributes to distance estimation even when visual information is available (Ellard \& Wagar, 2008). It is interesting that PD errors resemble those of young healthy 
adults when performing distance estimation using a cue-conflict paradigm (i.e., the experimenters provide conflicting locomotor and visual input to locate the target) (Ellard \& Wagar, 2008). Results from a recent study show that when participants were trained with the locomotor target which was further away than visual target, participants walked further than the visual distance on test trials. Similarly when trained on locomotor targets that were closer than visual targets, participants stopped earlier during the test trials. This study suggests that relationships between viewed egocentric targets and actions to walk are plastic, and also highlights the significant contribution of proprioceptive information during locomotion, and its weight when computing distance travelled to a target. Another alternative explanation might be that vision and/or proprioception requires more time for processing and during movement those with PD are unable to accurately process their sensory information fast enough to update and accurately track their movements through an environment. Previous studies have provided evidence that PD participants require a longer time to process properties of a stimulus and therefore have slower perceptual speed (Johnson et al., 2004). If this was the case, one might expect to see more accurate estimates if we slowed PD patients further during the perception with motion condition. Another possible explanation for the current results may be that individuals with PD have a visual motion processing deficit, which has been previously suggested (Castelo-Branco et al., 2009; Silva et al., 2005), such that during movement those with PD are unable to track the visual cues (i.e., optic flow) to compute the distance they have travelled. The current study was able to rule out this hypothesis by testing participants in a passive motion condition (i.e., pushed in a wheelchair) as well as in virtual reality where visual motion was simulated without vestibular information. During passive motion (wheelchair condition), both groups did not differ in judgment accuracy demonstrating that when all other sensory cues are available, and proprioception is limited, PD patients are able to estimate distance with motion as accurately as healthy control 
participants suggesting perhaps that visual motion stimuli engage neural circuits that are less affected by PD (Majsak, Kaminski, Gentile, \& Flanagan, 1998; Majsak, Kaminski, Gentile, \& Gordon, 2008).

Furthermore, during the simulation of forward walking, individuals with PD performed slightly better than control participants at estimating distance. These findings support previous hypotheses that individuals with PD are heavily dependent on vision in order to compensate for a proprioceptive deficit (Almeida et al., 2005; Jacobs \& Horak, 2006; Keijsers et al., 2005). Those with PD may not find the simulated movement condition (which is solely visual input and lacks proprioceptive information about movement) to be as unnatural or difficult as healthy control participants, since this experience may be similar to the input they receive during normal movement.

It should be noted that indeed both groups did increase their judgment errors during simulated movement. This is a common finding for the magnitude of error to increase in a simulated environment when compared to performance in physical reality. In fact, it is a characteristic finding that participants in VR consistently fall systematically short of true travel distances (Frenz \& Lappe, 2005; Harris et al., 2000; Wang et al., 2011). Previous literature has pointed out that virtual reality changes perception-action coupling by two respects (Wang et al., 2011). First, virtual reality fails to provide sufficient depth perception making it difficult to precisely locate the target position. Secondly, the performer in VR obtains visual feedback of movements that do not coincide with proprioceptive feedback, making the task feel unnatural. Research has also shown that individuals are more sensitive to motion in depth optic flow when combined with complementary vestibular input (which was the case during the wheelchair condition). This is another possible explanation for performance differences between the real world and simulated passive motion conditions (Edwards, O'Mahony, Ibbotson, \& Kohlhagen, 2010). Harris and colleagues used VR to investigate contributions of optic flow and vestibular information to estimate the distance travelled passively, and 
found that since participants did not receive proprioceptive information they found larger errors in estimations similar to the current findings in this study (Harris et al., 2000).

Taken together, these findings support the notion that a proprioceptive deficit is an underlying contributor to movement impairments in $\mathrm{PD}$, specifically during locomotion through an environment. From these findings, there was no evidence that a visual perceptual deficit contributed to impaired depth perception since PD participants did not differ from HC participants during the static estimation tasks. However, this study did not examine radial error, so it is not possible to rule out an argument for compression of space in one hemi field as seen in previous studies (Lee et al., 2001). In addition, the current study found little evidence of a visual motion processing deficit previously proposed (Castelo-Branco et al., 2009; Silva et al., 2005). However, the two visual motion tasks used in this study were specifically relevant to the role of optic flow in estimating distance, and as such were not as sensitive as many tasks previously used in the literature to detect it visual motion processing deficits.

\subsubsection{Dopaminergic Contributions}

The current study aimed to clarify the role of dopaminergic medication in sensory processing, and to determine whether dopaminergic replacement therapy was able to help with sensory-perceptual deficits in those with PD. Statistically, individuals with PD "OFF" dopaminergic medication did not differ from their performance "ON" medication across all conditions. It appears that dopaminergic medication did not influence PD participants' judgments during the static or visual motion conditions. However, slight improvements were seen when PD participants were ON their medications specifically during the motion condition. Interestingly, PD-ON when compared to HC participants did not differ significantly during walking to the remembered target. Interpretation of these findings is quite difficult since PD do not significantly differ from OFF to ON, and PD-ON do not differ from HC, however PD-OFF and HC are significantly different. Similar results have been found in previous 
studies when examining dopaminergic contributions to proprioception (Almeida et al., 2005). A common explanation for a lack of difference between PD-ON and $\mathrm{HC}$ is the increase in variability when $\mathrm{PD}$ are 'ON' their medication, however the findings in this study show that variability in their judgments did not change when PD were 'OFF' to 'ON' their regular dopaminergic medication. Jacobs and Horak (2006) also found that dopaminergic medication did not consistently improve the accuracy of stepping to a target and proposed that the dopaminergic circuits within the BG may not be responsible for proprioceptive-motor deficits. If the BG were directly responsible for proprioceptivemotor deficits, then a homogeneous effect of dopaminergic medication would be expected since the loss of nigro-striatal dopaminergic cells ought to be robust for all PD. Instead, researchers suggest that the supplementary motor area may be involved which degrades in PD, but only responds to dopamine replacement in those with severe impairment in those regions which involve proprioceptive processing and motor execution (Jacobs \& Horak, 2006; Mongeon et al., 2009). Alternatively previous studies have also suggested that dopaminergic medication worsens proprioceptive deficits in PD (Mongeon et al., 2009; O'Suilleabhain et al., 2001). The current study does not provide support for this hypothesis either, since performance ' $\mathrm{ON}$ ' medication did not get worse during walking conditions but instead slightly improved. Further research needs to closely examine the relationship between dopaminergic replacement therapy and sensorimotor deficits in PD to gain a better understanding of how to rehabilitate movement impairments seen in PD during locomotion through an environment.

\subsubsection{Considerations/ Limitations}

The findings from this study cannot be explained by differences between groups in spatial working memory since differences were not found between PD and $\mathrm{HC}$ across all conditions which required participants to hold the location of the target in memory for a period of time in order to give their judgment. Furthermore, PD participants were equivalent to healthy age-matched control participants 
on spatial working memory scores using the Corsi block tapping task. In addition, to rule out differences related to motor deficits, a crude measure of movement time during the walking conditions was collected but did not show significant differences between the groups during the walking task. It should be noted however, that there were characteristic velocity differences when measuring their regular velocity at pre-test between groups. Motor planning deficits and time perception deficits were taken into consideration as a potential explanation for the results found in this study. In order to confidently rule out these impairments as an explanation for the underestimations during the walking task in $\mathrm{PD}$, all participants completed a series of motor imagery trials comparing the time participants took to walk to a target, and the time it took them to imagine themselves walking to the same target (Bakker et al., 2007; Snijders et al., 2011). If motor planning or time perception was indeed a contributor to the underestimations seen during the walking trials, one would expect that those with PD would have lower accuracy in imagining their movements to a target, than healthy age-matched control, but no such difference was found. Finally, gender differences are well-documented in studies of distance estimation, and often demonstrate than women show less accurate performance than men (Ellard \& Shaughnessy, 2003). The current study was unable to match groups with gender, such that the PD group was primarily males and the healthy-age matched control participants were primarily females. Therefore, estimates of differences between PD and $\mathrm{HC}$ participants in the current study may be conservative all other considerations notwithstanding; the male-dominated PD group would be expected to perform better than the femaledominated healthy control group.

\subsubsection{Future Implications}

The findings of this study emphasize the important contribution of proprioceptive processing and integration deficits to movement impairments in PD. The current study demonstrated that sensory perceptual deficits exist in PD. It remains unclear whether these deficits result from poor 
proprioceptive processing or from integration of proprioceptive and visual information. Further research is needed to clarify this relationship. In addition, it remains unclear whether those with PDFOG exhibit similar deficits as seen in this study, or if this subtype of severe PD includes different perceptual deficits that may be involved in triggering freezing behaviour. Further research should investigate whether PD-FOG show characteristic proprioceptive deficits during locomotion and whether they have more severe or additional perceptual impairments that may underlie FOG episodes. The current study presents an ecologically valid protocol since participants completed all conditions with their eyes open, just as they would during their normal daily behaviours. Manipulation of cognitive load during an obstacle avoidance or navigational task may further demonstrate situations in which individuals are required to relying more heavily on proprioception to guide their movements, which might result in inaccurate movement patterns culminating in trips or falls. Furthermore, virtual environments should be considered as an excellent tool for further investigation of visual and proprioceptive integration deficits. The current study demonstrated that individuals with PD can feel immersed in a virtual environment, and that virtual reality provides the freedom to safely manipulate sensory cues and their accuracy in order to train PD to rely more on locomotor information. This has been shown in previous research with young adults which demonstrated considerable plasticity in the relationship between visual and proprioceptive information during a walking task(Ellard \& Wagar, 2008).

\subsubsection{Conclusion}

To our knowledge this is the first study to compare perception of space and movement in PD across all sensory modalities, both ON and OFF dopaminergic medication. The current study confirmed that participants with PD have impairments in perception of self-motion which contribute to inaccurate estimates of egocentric distance walking, and may lead to inaccurate movements commonly seen in PD. Findings highlight that proprioceptive input is the main source of error that leads to motion 
perception deficits. However this study is unable to rule out visual-proprioceptive integration deficits. Dopaminergic medication does not seem to significantly improve self-motion deficits in PD, and therefore it remains unclear whether these deficits are caused by damage to the BG circuitry in PD. Future research should investigate the origins of self-motion impairments in PD and, more specifically, how they contribute to movement deficits such as trips, falls and FOG in order to develop rehabilitative strategies for these movement impairments that cannot be treated fully with dopaminergic replacement therapy. 


\section{References}

Adamovich, S. V., Berkinblit, M. B., Hening, W., Sage, J., \& Poizner, H. (2001). The interaction of visual and proprioceptive inputs in pointing to actual and remembered targets in Parkinson's disease. Neuroscience, 104(4), 1027-1041.

Almeida, Q. J., Frank, J. S., Roy, E. A., Jenkins, M. E., Spaulding, S., Patla, A. E., et al. (2005). An evaluation of sensorimotor integration during locomotion toward a target in Parkinson's disease. Neuroscience, 134(1), 283-293.

Almeida, Q. J., \& Lebold, C. A. (2010). Freezing of gait in Parkinson's disease: a perceptual cause for a motor impairment? Journal of Neurology Neurosurgery and Psychiatry, 81(5), 513-518.

Bakker, M., de Lange, F. P., Stevens, J. A., Toni, I., \& Bloem, B. R. (2007). Motor imagery of gait: a quantitative approach. Exp Brain Res, 179(3), 497-504.

Barlow, J. S. (1964). Inertial navigation as a basis for animal navigation. J Theor Biol, 6(1), 76-117.

Bigel, M. G., \& Ellard, C. G. (2000). The contribution of nonvisual information to simple place navigation and distance estimation: an examination of path integration. Can J Exp Psychol, $54(3), 172-185$.

Bloem, B. R., Hausdorff, J. M., Visser, J. E., \& Giladi, N. (2004). Falls and freezing of gait in Parkinson's disease: a review of two interconnected, episodic phenomena. Mov Disord, 19(8), 871-884.

Bodis-Wollner, I. G., \& Paulus, W. (1999). Visual and visual cognitive dysfunction in Parkinson's disease: spatial and chromatic vision. Parkinson's Disease, 80, 383-388. 
Boecker, H., Ceballos-Baumann, A., Bartenstein, P., Weindl, A., Siebner, H. R., Fassbender, T., et al. (1999a). Sensory processing in Parkinson's and Huntington's disease - Investigations with 3D (H2O)-O-15-PET. Brain, 122, 1651-1665.

Boecker, H., Ceballos-Baumann, A., Bartenstein, P., Weindl, A., Siebner, H. R., Fassbender, T., et al. (1999b). Sensory processing in Parkinson's and Huntington's disease: investigations with 3D H(2)(15)O-PET. Brain, 122 ( Pt 9), 1651-1665.

Bremmer, F., \& Lappe, M. (1999). The use of optical velocities for distance discrimination and reproduction during visually simulated self motion. Exp Brain Res, 127(1), 33-42.

Castelo-Branco, M., Mendes, M., Silva, F., Massano, J., Januario, G., Januario, C., et al. (2009). Motion integration deficits are independent of magnocellular impairment in Parkinson's disease. Neuropsychologia, 47(2), 314-320.

Contreras-Vidal, J. L. (1999). The gating functions of the basal ganglia in movement control. Prog Brain Res, 121, 261-276.

Cowie, D., Limousin, P., Peters, A., \& Day, B. L. (2010). Insights into the neural control of locomotion from walking through doorways in Parkinson's disease. Neuropsychologia, 48(9), 2750-2757.

Durgin, F. H., Pelah, A., Fox, L. F., Lewis, J., Kane, R., \& Walley, K. A. (2005). Self-motion perception during locomotor recalibration: more than meets the eye. J Exp Psychol Hum Percept Perform, 31(3), 398-419.

Edwards, M., O'Mahony, S., Ibbotson, M. R., \& Kohlhagen, S. (2010). Vestibular stimulation affects optic-flow sensitivity. Perception, 39(10), 1303-1310.

Ellard, C. G., \& Shaughnessy, S. C. (2003). A comparison of visual and nonvisual sensory inputs to walked distance in a blind-walking task. Perception, 32(5), 567-578. 
Ellard, C. G., \& Wagar, L. S. (2008). Plasticity of the association between visual space and action space in a blind-walking task. Perception, 37(7), 1044-1053.

Frenz, H., \& Lappe, M. (2005). Absolute travel distance from optic flow. Vision Res, 45(13), 16791692.

Giladi, N., Treves, T. A., Simon, E. S., Shabtai, H., Orlov, Y., Kandinov, B., et al. (2001). Freezing of gait in patients with advanced Parkinson's disease. J Neural Transm, 108(1), 53-61.

Glasauer, S., Amorim, M. A., Vitte, E., \& Berthoz, A. (1994). Goal-directed linear locomotion in normal and labyrinthine-defective subjects. Exp Brain Res, 98(2), 323-335.

Graziano, M. S., \& Gross, C. G. (1993). A bimodal map of space: somatosensory receptive fields in the macaque putamen with corresponding visual receptive fields. Exp Brain Res, 97(1), 96109.

Harris, L. R., Jenkin, M., \& Zikovitz, D. C. (2000). Vestibular capture of the perceived distance of passive linear self motion. Arch Ital Biol, 138(1), 63-72.

Houk, J. C., \& Wise, S. P. (1995). Distributed modular architectures linking basal ganglia, cerebellum, and cerebral cortex: their role in planning and controlling action. Cereb Cortex, $5(2), 95-110$.

Jacobs, J. V., \& Horak, F. B. (2006). Abnormal proprioceptive-motor integration contributes to hypometric postural responses of subjects with Parkinson's disease. Neuroscience, 141(2), 999-1009.

Johnson, A. M., Almeida, Q. J., Stough, C., Thompson, J. C., Singarayer, R., \& Jog, M. S. (2004). Visual inspection time in Parkinson's disease: deficits in early stages of cognitive processing. Neuropsychologia, 42(5), 577-583. 
Keijsers, N. L., Admiraal, M. A., Cools, A. R., Bloem, B. R., \& Gielen, C. C. (2005). Differential progression of proprioceptive and visual information processing deficits in Parkinson's disease. Eur J Neurosci, 21(1), 239-248.

Klockgether, T., Borutta, M., Rapp, H., Spieker, S., \& Dichgans, J. (1995). A defect of kinesthesia in Parkinson's disease. Mov Disord, 10(4), 460-465.

Lappe, M., \& Frenz, H. (2009). Visual estimation of travel distance during walking. Experimental Brain Research, 199(3-4), 369-375.

Lappin, J. S., Shelton, A. L., \& Rieser, J. J. (2006). Environmental context influences visually perceived distance. Percept Psychophys, 68(4), 571-581.

Lee, A. C., Harris, J. P., Atkinson, E. A., \& Fowler, M. S. (2001). Disruption of estimation of bodyscaled aperture width in Hemiparkinson's disease. Neuropsychologia, 39(10), 1097-1104.

Loomis, J. M., Da Silva, J. A., Fujita, N., \& Fukusima, S. S. (1992). Visual space perception and visually directed action. J Exp Psychol Hum Percept Perform, 18(4), 906-921.

Loomis, J. M., DaSilva, J. A., Philbeck, J. W., \& Fukusima, S. S. (1996). Visual perception of location and distance. Current Directions in Psychological Science, 5(3), 72-77.

Majsak, M. J., Kaminski, T., Gentile, A. M., \& Flanagan, J. R. (1998). The reaching movements of patients with Parkinson's disease under self-determined maximal speed and visually cued conditions. Brain, 121 ( Pt 4), 755-766.

Majsak, M. J., Kaminski, T., Gentile, A. M., \& Gordon, A. M. (2008). Effects of a moving target versus a temporal constraint on reach and grasp in patients with Parkinson's disease. Exp Neurol, 210(2), 479-488.

Martens, K. A., \& Almeida, Q. J. (2011). Dissociating between sensory and perceptual deficits in PD: More than simply a motor deficit. Mov Disord. 
Maschke, M., Gomez, C. M., Tuite, P. J., Pickett, K., \& Konczak, J. (2006). Depth perception in cerebellar and basal ganglia disease. Exp Brain Res, 175(1), 165-176.

Mongeon, D., Blanchet, P., \& Messier, J. (2009). Impact of Parkinson's disease and dopaminergic medication on proprioceptive processing. Neuroscience, 158(2), 426-440.

Mosimann, U. P., Mather, G., Wesnes, K. A., O'Brien, J. T., Burn, D. J., \& McKeith, I. G. (2004). Visual perception in Parkinson disease dementia and dementia with Lewy bodies. Neurology, 63(11), 2091-2096.

Nagy, A., Eordegh, G., Paroczy, Z., Markus, Z., \& Benedek, G. (2006). Multisensory integration in the basal ganglia. Eur J Neurosci, 24(3), 917-924.

O'Suilleabhain, P., Bullard, J., \& Dewey, R. B. (2001). Proprioception in Parkinson's disease is acutely depressed by dopaminergic medications. J Neurol Neurosurg Psychiatry, 71(5), 607610.

Redlick, F. P., Jenkin, M., \& Harris, L. R. (2001). Humans can use optic flow to estimate distance of travel. Vision Res, 41(2), 213-219.

Rektor, I., Bares, M., Kanovsky, P., Brazdil, M., Klajblova, I., Streitova, H., et al. (2004). Cognitive potentials in the basal ganglia-frontocortical circuits. An intracerebral recording study. Exp Brain Res, 158(3), 289-301.

Rickards, C., \& Cody, F. W. (1997). Proprioceptive control of wrist movements in Parkinson's disease. Reduced muscle vibration-induced errors. Brain, 120 ( Pt 6), 977-990.

Rieser, J. J., Pick, H. L., Jr., Ashmead, D. H., \& Garing, A. E. (1995). Calibration of human locomotion and models of perceptual-motor organization. J Exp Psychol Hum Percept Perform, 21(3), 480-497.

Schneider, J. S., Diamond, S. G., \& Markham, C. H. (1987). Parkinson's disease: sensory and motor problems in arms and hands. Neurology, 37(6), 951-956. 
Schrader, C., Peschel, T., Dauper, J., Rollnik, J. D., Dengler, R., \& Kossev, A. R. (2008). Changes in processing of proprioceptive information in Parkinson's disease and multiple system atrophy. Clin Neurophysiol, 119(5), 1139-1146.

Silva, M. F., Faria, P., Regateiro, F. S., Forjaz, V., Januario, C., Freire, A., et al. (2005). Independent patterns of damage within magno-, parvo- and koniocellular pathways in Parkinson's disease. Brain, 128(Pt 10), 2260-2271.

Snijders, A. H., Leunissen, I., Bakker, M., Overeem, S., Helmich, R. C., Bloem, B. R., et al. (2011). Gait-related cerebral alterations in patients with Parkinson's disease with freezing of gait. Brain, 134(Pt 1), 59-72.

Sun, H. J., Campos, J. L., Young, M., Chan, G. S., \& Ellard, C. G. (2004). The contributions of static visual cues, nonvisual cues, and optic flow in distance estimation. Perception, 33(1), 49-65.

Thomson, J. A. (1983). Is continuous visual monitoring necessary in visually guided locomotion? J Exp Psychol Hum Percept Perform, 9(3), 427-443.

Trick, G. L., Kaskie, B., \& Steinman, S. B. (1994). Visual impairment in Parkinson's disease: deficits in orientation and motion discrimination. Optom Vis Sci, 71(4), 242-245.

Wang, C. Y., Hwang, W. J., Fang, J. J., Sheu, C. F., Leong, I. F., \& Ma, H. I. (2011). Comparison of virtual reality versus physical reality on movement characteristics of persons with Parkinson's disease: effects of moving targets. Arch Phys Med Rehabil, 92(8), 1238-1245.

Wright, W. G., Gurfinkel, V., King, L., \& Horak, F. (2007). Parkinson's disease shows perceptuomotor asymmetry unrelated to motor symptoms. Neurosci Lett, 417(1), 10-15.

Zia, S., Cody, F., \& O'Boyle, D. (2000). Joint position sense is impaired by Parkinson's disease. Annals of Neurology, 47(2), 218-228. 